\title{
Radiation dosimetry of 18F-FDG PET/CT: incorporating exam-specific parameters in dose estimates
}

\author{
Brian Quinn 1*, Zak Dauer ${ }^{1}$, Neeta Pandit-Taskar ${ }^{2}$, Heiko Schoder $^{2}$ and Lawrence T. Dauer ${ }^{1,2}$
}

\begin{abstract}
Background: Whole body fluorine-18 fluorodeoxyglucose positron emission tomography/computed tomography (PET/CT) is the standard of care in oncologic diagnosis and staging, and patient radiation dose must be well understood to balance exam benefits with the risk from radiation exposure. Although reference PET/CT patient doses are available, the potential for widely varying total dose prompts evaluation of clinic-specific patient dose. The aims of this study were to use exam-specific information to characterize the radiation dosimetry of PET/CT exams that used two different $C T$ techniques for adult oncology patients and evaluate the practicality of employing an exam-specific approach to dose estimation.
\end{abstract}

Methods: Whole body PET/CT scans from two sets of consecutive adult patients were retrospectively reviewed. One set received a PET scan with a standard registration CT and the other a PET scan with a diagnostic quality CT. PET dose was calculated by modifying the standard reference phantoms in OLINDA/EXM 1.1 with patient-specific organ mass. CT dose was calculated using patient-specific data in ImPACT. International Commission on Radiological Protection publication 103 tissue weighting coefficients were used for effective dose.

Results: One hundred eighty three adult scans were evaluated (95 men, 88 women). The mean patient-specific effective dose from a mean injected 18F-FDG activity of $450 \pm 32 \mathrm{MBq}$ was $9.0 \pm 1.6 \mathrm{mSv}$. For all standard PET/CT patients, mean effective mAs was $39 \pm 11 \mathrm{mAs}$, mean CT effective dose was $5.0 \pm 1.0 \mathrm{mSv}$ and mean total effective dose was $14 \pm 1.3 \mathrm{mSv}$. For all diagnostic PET/CT patients, mean effective mAs was $120 \pm 51 \mathrm{mAs}$, mean CT effective dose was $15.4 \pm 5.0 \mathrm{mSv}$ and mean total effective dose was $24.4 \pm 4.3 \mathrm{mSv}$. The five organs receiving the highest organ equivalent doses in all exams were bladder, heart, brain, liver and lungs.

Conclusions: Patient-specific parameters optimize the patient dosimetry utilized in the medical justification of whole body PET/CT referrals and optimization of PET and CT acquisition parameters. Incorporating patient-specific data into dose estimates is a worthwhile effort for characterizing patient dose, and the specific dosimetric information assists in the justification of risk and optimization of PET/CT.

Keywords: PET/CT, CT, Radiation exposure, Effective dose, 18F-FDG

\footnotetext{
* Correspondence: quinnb@mskcc.org

Brian Quinn is the first author.

${ }^{1}$ Department of Medical Physics, Box 84, Memorial Sloan Kettering Cancer

Center, 1275 York Avenue, New York, NY 10065, USA

Full list of author information is available at the end of the article
} 


\section{Background}

Positron emission tomography/computed tomography (PET/CT) has become an indispensable imaging modality for the diagnosis, staging and monitoring of therapy response of a broad range of malignancies [1, 2]. PET/ CT is a valuable tool in oncology due to the combined metabolic and morphological information provided. The PET emission scan provides physiological information using a set of detectors that are independent of CT transmission detection, and although two independent PET and CT images may be coregistered to form a single image, the two scans are most accurately coupled when both are acquired during the same exam using a combined PET and CT scanner. For this reason, and because a built-in $\mathrm{CT}$ provides other conveniences such as attenuation correction, most modern PET scanners are dual PET/CT units. Regardless of acquisition circumstances, the patient dose from PET and the patient dose from CT in PET/CT exams are first estimated separately in different ways, and then combined to give a total whole body radiation dose. Referral for PET/CT studies must be justified in each case as a first general principle of radiological protection [3]. Optimization, or ensuring that the diagnostic information is as high as reasonably achievable while maintaining radiation doses as low as reasonably achievable, is the second general principle in radiologic protection according to the International Committee on Radiological Protection (ICRP) [4]. Additionally, increased awareness of the risk of exposure to ionizing radiation has resulted in efforts to minimize radiation dose incurred during $\mathrm{x}$-ray and nuclear medical imaging tests [5]. Implementation of any dose saving strategies depends critically on accurate dose measurement/dosimetry to maximize the benefit/risk ratio from imaging tests [6].

A reliable method of estimating patient dose, which includes appropriate units of dose measurement and appropriate calculation methods, is important for any exam involving ionizing radiation. As Stabin stated, the biokinetic model used to calculate the dose is one of the major uncertainties in the evaluation of radiation doses for radiopharmaceuticals [7]. If careful patient specific dosimetry is performed, with attention paid to accurate measurement of individual organ volumes, many of the biokinetic model uncertainties can be minimized, and the total uncertainty in the individual dose estimate can be reduced to perhaps $\pm 10 \%-20 \%[7]$. Effective dose is a parameter that allows a meaningful comparison of the radiation dose from the radiopharmaceutical and the $\mathrm{x}$ ray portions of a PET/CT scan. Effective dose is not directly measured, rather it is calculated based on equivalent doses to organs and the radiosensitivities of the organs. Effective dose is commonly used when evaluating relative biologic risk (4), and the Monte Carlo-based organ dose coefficients should not be used to calculate effective dose for individual patients $[6,8]$. The International Organization for Medical Physics, ICRP, Health Physics Society and American Association of Physicists in Medicine (AAPM) all have issued policy statements that note the dangers of extrapolating biological risk estimates for radiation doses less than $100 \mathrm{mSv}$ [6, 9-11]. The establishment and use of risk coefficients to estimate public health determinants from individual or population exposures must be considered in the context of uncertainties in the estimates [12]. These uncertainties include dosimetric uncertainties, epidemiological and methodological uncertainties, uncertainties from low statistical power and precision in epidemiology studies of radiation risk, uncertainties in modeling risk data, generalization of risk estimates across different populations and dose rates, as well as reliance on epidemiological studies on observational rather than experimental data [13]. Uncertainties in such risk estimates have been suggested as being up to a factor of 3 lower or higher than the value itself [14]. These rather large uncertainties cause predictions of radiation induced cancers or detriment to be susceptible to biases and confounding influences that are unidentifiable. With due recognition of the limitations imposed by the uncertainties inherent in correlating dose to risk, effective dose is a useful approximation for relating patient dose from internal and external sources, evaluating population characteristics and evaluating optimization efforts. Organ doses from PET are estimated based on the injected activity, while organ doses from CT are estimated based on scannerspecific monte carlo simulations or the dose parameter reported in the exam dose report. Both approaches can be made to more accurately reflect the actual patient dose by including scanner- and patient-specific factors.

Without specific information, risk evaluations may be based on reference or literature values that must be carefully chosen if used for risk evaluation. The International Atomic Energy Agency's "Radiation Protection of Patients" (RPOP) is a reputable online reference resource that provides information for health healthcare professionals, member states and the general public to achieve safer use of radiation [15]. In the absence of specifics, reference values can overgeneralize and can result in conservative overestimates of dose in an attempt to account for many variables. Such generalization is inherent in non-specific reference values for PET/CT exams because of the number of PET and $\mathrm{CT}$ variables contributing to overall dose. CT technique differs greatly if it is just for attenuation correction or if it is for diagnosticquality images. Attenuation correction can be performed with CT currents as low as $10 \mathrm{~mA}$ but most institutions use CT currents of 40-80 mA [16]. Further, an oncology center, for example, may have an intermediate CT 
technique that provides images with better image quality than a technique only for attenuation correction but lower image quality than a diagnostic CT technique. PET dose varies primarily with injected activity, but like CT dose varies with patient size. The dependency of total dose on many factors warrants a critical review of general reference values and suggests the importance of specific data.

Several approaches to PET/CT dose estimation utilize exam-specific parameters. Dose Length Product (DLP) is a standardized CT dose parameter that is established for each CT scanner using a phantom, equal to CTDIvol multiplied by the length of the scan. DLP can be related to effective dose by multiplying DLP by a patient-specific factor, or scanner-specific monte carlo simulation data can be employed to estimate CT dose $[17,18]$. ImPACT method calculates organ dose from CT and uses simplified stylized anatomic models that are anatomically crude but widely used for practical applications with the standard mathematic representations of the reference man and other representative phantoms in radiation protection, nuclear medicine, and medical imaging [19-21]. Radiopharmaceutical organ dose is estimated utilizing conversion coefficients and injected activity, such as those in ICRP 106. Effective dose is typically calculated from both PET and CT organ dose utilizing tissue-weighting factors, such as those in ICRP 103.

In these ways, effective dose from both PET and CT both are calculated and combined to represent a whole body burden. This combined whole body burden is useful in evaluating both the relative and absolute benefit of the combined scan. Although PET/CT is widely performed, published patient dose data is as varied as the parameters determining the dose and often focuses on a narrow patient population [22-31]. The potential for variation in PET scan practices and CT techniques is especially apparent when one considers the wide range of scanner makes \& models, injected activity and patient populations in different countries. Many clinic-specific elements that influence radiation dose can be incorporated into the dose estimate to make the dose more specific to the patient.

The aim of the present study was to characterize the radiation dosimetry of two types of routine whole body $\mathrm{PET} / \mathrm{CT}$ protocols at our institution using patient-specific data and commonly available dose estimation techniques. We compare the results of using patient-specific data with reference and published values and ascertain the utility of such data in the evaluation of risk/benefit for justification and protocol optimization in routine clinical utilization.

\section{Methods}

\section{Study population}

Institutional review board approval with waiver of patient informed consent was obtained to perform a retrospective study of clinically indicated whole body PET/
CT scans performed on consecutive adult oncology patients at Memorial Sloan Kettering Cancer Center from January 2010 through December 2011. Patient consent was not required.

\section{Patient population data}

Consecutive adult patients who had undergone PET/CT with either standard registration or full dose diagnostic quality CT techniques for a variety of oncological indications, were identified. Patient demographics are shown in Table 1.

\section{Exam data}

For each PET exam performed, the amount of 18F-FDG administered was obtained from the medical records. For each CT study, image data was reviewed to determine the body region that was being examined, and this was repeated for each CT series. The following parameters for each series were extracted from the DICOM headers, dose reports and scan scout images for later organ equivalent dose and effective dose calculation: (a) $\mathrm{kVp}$, (b) $\mathrm{mA}$, (c) beam collimation, (d) rotation time, (e) pitch, (f) volume CT dose index (CTDIvol) and (g) dose length product (DLP).

\section{F-FDG PET/CT exam}

All whole-body PET/CT patients fast for at least $6 \mathrm{~h}$, blood glucose concentration is determined before the injection of the radiopharmaceutical. A nominal injection activity of $12 \mathrm{mCi}(444 \mathrm{MBq})$ is prescribed in our clinic, and $+/-10 \%$ of the prescribed activity is actually injected intravenously into the patient. The actual injected activity is recorded for each patient. Following an approximately $60 \mathrm{~min}$ uptake time, PET/CT exams are performed on General Electric (GE) scanners. Data for this study were recorded from patients scanned on Discovery 690 DSTE PET/CT, which utilizes a GE Lightspeed 16 CT scanner (GE Healthcare, Milwaukee, Wis). Tube current modulation is utilized for all CT acquisitions except the "scout" scan, which uses a fixed technique of $120 \mathrm{kVp}, 10 \mathrm{~mA}$, pitch 3 and rotation time $0.5 \mathrm{~s}$. The first part of the exam sequence is acquisition of a scout CT image used to select the desired anatomy to be scanned and establish bed positions for PET acquisition. The technologist selects from base of the skull to

Table 1 Patient demographics

\begin{tabular}{llll}
\hline & $\begin{array}{l}\text { No. } \\
\text { patients }\end{array}$ & Age & Body mass index \\
& & Yrs & \\
\hline Male & 95 & $53.7 \pm 18.1(18.8-90.2)$ & $26.2 \pm 4.8(15.1-46.1)$ \\
Female & 88 & $52.1 \pm 20.3(18.4-96.7)$ & $26.6 \pm 7.8(15.6-71.5)$ \\
Male and female & 183 & $52.3 \pm 19.3(18.4-96.7)$ & $26.4 \pm 6.4(15.1-71.5)$ \\
\hline
\end{tabular}

All data are mean \pm standard deviation (range) 
mid thighs for body PET/CT scans. Next a CT scan is acquired using either the standard or the diagnostic $\mathrm{CT}$ technique, depending on the required patient specific protocols completed by the radiologist. The technique for all standard PET/CT exams in this study is $120 \mathrm{kVp}$, auto-mA, rotation time $0.8 \mathrm{sec}$, slice thickness $3.75 \mathrm{~mm}$, collimator width 10 or $40 \mathrm{~mm}$, pitch 1.75 . The technique for all PET/CT exams with a diagnostic CT is $120 \mathrm{kVp}$, auto-mA, rotation time $0.6 \mathrm{~s}$, slice thickness $5.00 \mathrm{~mm}$, collimator width 20 or $40 \mathrm{~mm}$, pitch 1.375 . CT techniques are summarized in Table 2 .

$\mathrm{CT}$ is followed immediately by PET acquisition with a 3-min emission acquisition time per bed position, a 15.3-cm axial field of view per bed position with a $23 \%$ bed overlap.

\section{Internal radiation-absorbed dose assessment}

The OLINDA\EXM code (version 1.1, Vanderbilt University, Nashville, TN, USA) was used to determine the organ equivalent dose and effective dose from each PET series with patient-specific parameters [32]. The biokinetic model parameters as defined in Bolch et al (2009) and specifically identified in ICRP Publication 106 for 18F-FDG were used as input factors for OLINDA [33, 34]. The ICRP biokinetic model for 18F-FDG was derived from data in Hayes et al and Deloar et al [35, 36]. The OLINDA code also allows for the modification of standard reference phantoms to more closely represent patientspecific factors, such as patient weight and corresponding organ mass. We used the standard anthropomorphic models as well as models modified to represent patients weight and height (i.e., organ sizes of the phantom models used by OLINDA were modified to reflect the patientspecific mass of the organ as described by Marine et al and Clark et al [37-39]. Modified adult male, adult female models in OLINDA's phantom library were utilized to generate patient-specific organ equivalent dose, and then tissue weighting factors from ICRP Publication 103 were used to generate patient-specific effective dose conversion factor $(\mathrm{mSv} / \mathrm{MBq})$ [6]. These factors were multiplied by injected activity (MBq) for each PET study to obtain an estimation of effective dose.

\section{External radiation-absorbed dose assessment}

Effective dose from CT examination was estimated using the CT-specific method using the ImPACT spreadsheet, employing ICRP 103 weighting factors [17].

\section{Statistical analysis}

Descriptive and summary statistics were performed with a spreadsheet application (Excel 2007, Microsoft, Redmond, WA). Statistical analysis included average, median, standard deviation, minimum, maximum and range.

\section{Results}

Study population

Of the total 183 PET/CT scans evaluated, 95 (52\%) were performed on male patients and 88 (48 \%) were performed on female patients. Forty-six male patients and 39 female patients received the standard CT technique. Forty-nine male patients and 49 female patients received the diagnostic CT technique. Subjects ranged in age from 18 to 96 years (mean \pm standard deviation (SD) $52.6 \pm 18.2$ years). The subjects' weight ranged (mean \pm SD) from 42.0 to $182 \mathrm{~kg}(74.9 \pm 17.7 \mathrm{~kg})$, height ranged (mean \pm SD) from 120 to $198 \mathrm{~cm}(168 \pm 10.3 \mathrm{~cm})$, and BMI ranged (mean \pm SD) from 15.1 to $71.5(26.4 \pm 6.4)$.

\section{F-FDG}

The mean \pm SD (range) 18F-FDG injected activity for all patients was $454 \pm 33.3 \mathrm{MBq}(152$ to $488 \mathrm{MBq})$.

\section{CT techniques}

The mean $\pm \mathrm{SD}$ (range) effective $\mathrm{mAs}$ of the standard CT for all patients was $39.0 \pm 11.2 \mathrm{mAs}$ (27.4 to 69.4 mAs), $38.6 \pm 10.0 \mathrm{mAs}$ (27.4 to $69.4 \mathrm{mAs}$ ) for male patients and $39.4 \pm 12.4 \mathrm{mAs}$ (27.4 to $69.4 \mathrm{mAs}$ ) for female patients.

The mean \pm SD (range) effective mAs of the diagnostic CT for all patients was $119 \pm 50.6 \mathrm{mAs}$ (27.4 to $231 \mathrm{mAs}$ ),

Table 2 CT technique summary

\begin{tabular}{|c|c|c|c|c|c|c|c|c|c|c|}
\hline $\begin{array}{l}\mathrm{CT} \text { technique } \\
\text { description }\end{array}$ & $\begin{array}{l}\text { Tube } \\
\text { potential } \\
(\mathrm{k} \vee p)\end{array}$ & $\begin{array}{l}\text { Tube current } \\
(\mathrm{mA})\end{array}$ & $\begin{array}{l}\text { Revolution time } \\
\text { (s) }\end{array}$ & $\begin{array}{l}\text { Collimator } \\
\text { width } \\
(\mathrm{mm})\end{array}$ & Pitch & Effective $m A s^{a}$ & $\begin{array}{l}\text { CTDIvol } \\
\text { (mGy) }\end{array}$ & $\begin{array}{l}\text { DLP } \\
(\mathrm{mGy} \mathrm{cm})\end{array}$ & $\begin{array}{l}\text { Effective Dose } \\
\text { (mSv) }\end{array}$ & $\begin{array}{l}\text { Effective dose } \\
\text { per unit mAs } \\
\text { (mSv/mAs) }\end{array}$ \\
\hline Standard male & 120 & $78 \pm 8.2$ & 0.8 & 10 or 40 & 1.75 & $38.6 \pm 10.0$ & $5.1 \pm 0.6$ & $464 \pm 86$ & $5.3 \pm 1.0$ & $0.142 \pm 0.026$ \\
\hline Standard female & 120 & $72 \pm 11$ & 0.8 & 10 or 40 & 1.75 & $39.4 \pm 12.4$ & $4.8 \pm 0.7$ & $407 \pm 73$ & $4.6 \pm 0.8$ & $0.124 \pm 0.028$ \\
\hline $\begin{array}{l}\text { Diagnostic } \\
\text { male }\end{array}$ & 120 & $236 \pm 86$ & 0.6 & 20 or 40 & 1.375 & $116 \pm 40.5$ & $12.1 \pm 2.8$ & $1185 \pm 249$ & $17.4 \pm 3.7$ & $0.164 \pm 0.063$ \\
\hline $\begin{array}{l}\text { Diagnostic } \\
\text { female }\end{array}$ & 120 & $223 \pm 109$ & 0.6 & 20 or 40 & 1.375 & $122 \pm 59.3$ & $10.2 \pm 3.9$ & $912 \pm 368$ & $13.4 \pm 5.5$ & $0.121 \pm 0.039$ \\
\hline
\end{tabular}

Data are the mean \pm standard deviation

${ }^{a}$ Effective $\mathrm{mAs}$ is calculated as [( $\mathrm{mA}^{*}$ tube rotation)/pitch] 
$116 \pm 40.5 \mathrm{mAs}$ (50.0 to $231 \mathrm{mAs}$ ) for male patients and $121.6 \pm 59.3 \mathrm{mAs}$ ( 27.1 to $231 \mathrm{mAs}$ ) for female patients.

CT techniques are summarized in Table 2.

\section{Radiation doses - 18F-FDG}

The five organs with the highest organ equivalent doses from 18F-FDG in all patients, in order of highest to lowest dose, were bladder, heart, brain, liver and lungs. 18F-FDG organ equivalent doses are summarized in Tables 3 and 4 .

Mean \pm SD (range) patient-specific effective dose from 18F-FDG calculated by OLINDA for all patients was $9.0 \pm 1.6 \mathrm{mSv}$ ( 3.4 to $13.6 \mathrm{mSv}$ ), $9.1 \pm 1.7 \mathrm{mSv}$ (5.4 to $12.8 \mathrm{mSv}$ ) for male and $10.0 \pm 1.5 \mathrm{mSv}$ (3.4 to $13.6 \mathrm{mSv}$ ) for female. Mean \pm SD (range) effective dose per unit injected activity for all patients was $0.0199 \pm$ $0.0032 \mathrm{mSv} / \mathrm{MBq}$ (0.0132 to $0.0291 \mathrm{mSv} / \mathrm{MBq})$. $18 \mathrm{~F}-$ FDG patient-specific effective doses are summarized in Table 5.

\section{Radiation doses - CT}

The mean \pm SD (range) effective dose for all patients from the $\mathrm{CT}$ portion of scans employing the standard technique was $5.0 \pm 1.0 \mathrm{mSv}$ ( 2.9 to $7.2 \mathrm{mSv}$ ), while it was $15.4 \pm 5.0 \mathrm{mSv}$ (5.4 to $27.8 \mathrm{mSv}$ ) for the scans employing a diagnostic technique.

The mean \pm SD (range) effective dose per unit effective $\mathrm{mAs}$ from all exams was $0.138 \pm 0.046 \mathrm{mSv} / \mathrm{mAs}(0.036$ to $0.445 \mathrm{mSv} / \mathrm{mAs}$ ) for all patients, from standard CT was $0.134 \pm 0.028 \mathrm{mSv} / \mathrm{mAs}$ ( 0.067 to $0.185 \mathrm{mSv} / \mathrm{mAs}$ ), and from diagnostic CT was $0.142 \pm 0.056 \mathrm{mSv} / \mathrm{mAs}$ (0.036 to $0.445 \mathrm{mSv} / \mathrm{mAs}$ ).

The mean \pm SD (range) effective dose per unit doselength product (DLP) from all exams was $0.018 \pm$ $0.046 \mathrm{mSv} / \mathrm{mGy}-\mathrm{cm}$ (0.036 to $0.445 \mathrm{mSv} / \mathrm{mGy}-\mathrm{cm})$ for all patients.

CT radiation doses are summarized in Table 6.

The five organs with the highest organ equivalent doses from standard CT in all patients, in order of highest to lowest dose, were thyroid, osteogenic cells, thymus, lungs

Table 3 Organ equivalent dose and effective dose from 18F-FDG PET/CT with standard CT to adult male and female patients

\begin{tabular}{|c|c|c|c|c|c|c|}
\hline \multirow[t]{2}{*}{ Organ } & \multicolumn{2}{|c|}{ Dose from 18F-FDG, mGy } & \multicolumn{2}{|c|}{ Dose from $C T, \mathrm{mGy}^{\mathrm{b}}$} & \multicolumn{2}{|c|}{ Total dose, mGy } \\
\hline & Male $(n=46)$ & Female $(n=39)$ & Male $(n=46)$ & Female $(n=39)$ & Male $(n=46)$ & Female $(n=39)$ \\
\hline Adrenals & $5.1 \pm 0.9$ & $6.0 \pm 1.0$ & $5.3 \pm 1.0$ & $4.6 \pm 0.8$ & $10.4 \pm 0.9$ & $10.6 \pm 0.9$ \\
\hline Brain & $15.9 \pm 3.2$ & $17.3 \pm 3.2$ & $6.0 \pm 1.0$ & $5.3 \pm 0.9$ & $21.9 \pm 2.8$ & $22.6 \pm 2.9$ \\
\hline Breasts & $3.6 \pm 0.7$ & $4.3 \pm 0.8$ & $4.9 \pm 0.9$ & $4.2 \pm 0.7$ & $8.4 \pm 0.8$ & $8.5 \pm 0.7$ \\
\hline Gallbladder wall & $5.4 \pm 1.0$ & $6.2 \pm 1.8$ & $5.6 \pm 1.0$ & $4.9 \pm 0.9$ & $11.1 \pm 0.9$ & $11.1 \pm 1.0$ \\
\hline Colon & $4.9 \pm 0.9$ & $5.9 \pm 1.0$ & $5.2 \pm 1.0$ & $4.6 \pm 0.8$ & $10.2 \pm 0.9$ & $10.5 \pm 0.9$ \\
\hline Small intestine & $5.1 \pm 0.9$ & $5.7 \pm 1.0$ & $5.2 \pm 1.0$ & $4.6 \pm 0.8$ & $10.3 \pm 0.9$ & $10.2 \pm 0.9$ \\
\hline Stomach wall & $4.7 \pm 0.9$ & $5.6 \pm 1.0$ & $5.7 \pm 1.0$ & $4.9 \pm 0.9$ & $10.3 \pm 0.9$ & $10.5 \pm 0.9$ \\
\hline Heart wall & $29.1 \pm 6.2$ & $35.4 \pm 6.9$ & $5.9 \pm 1.1$ & $5.2 \pm 0.9$ & $35.0 \pm 5.6$ & $40.6 \pm 6.5$ \\
\hline Kidneys & $4.5 \pm 0.8$ & $5.3 \pm 0.9$ & $5.8 \pm 1.1$ & $5.1 \pm 0.9$ & $10.3 \pm 0.9$ & $10.3 \pm 0.9$ \\
\hline Liver & $8.9 \pm 1.7$ & $11.0 \pm 2.0$ & $5.6 \pm 1.0$ & $4.8 \pm 0.8$ & $14.4 \pm 1.4$ & $15.8 \pm 1.7$ \\
\hline Lungs & $8.3 \pm 1.7$ & $10.0 \pm 1.8$ & $6.3 \pm 1.2$ & $5.4 \pm 1.0$ & $14.6 \pm 1.4$ & $15.4 \pm 1.5$ \\
\hline Muscle & $4.2 \pm 0.8$ & $5.0 \pm 0.9$ & $3.8 \pm 0.7$ & $3.3 \pm 0.6$ & $8.0 \pm 0.7$ & $8.3 \pm 0.7$ \\
\hline Ovaries/Testes ${ }^{c}$ & $4.6 \pm 0.8$ & $7.0 \pm 1.2$ & $1.1 \pm 0.2$ & $4.3 \pm 0.8$ & $5.6 \pm 0.8$ & $11.2 \pm 1.0$ \\
\hline Pancreas & $5.2 \pm 1.0$ & $6.2 \pm 1.0$ & $5.2 \pm 1.0$ & $4.5 \pm 0.8$ & $10.4 \pm 0.9$ & $10.7 \pm 0.9$ \\
\hline Red marrow & $4.2 \pm 0.9$ & $4.8 \pm 1.0$ & $4.4 \pm 0.8$ & $3.8 \pm 0.7$ & $8.5 \pm 0.8$ & $8.6 \pm 0.9$ \\
\hline Osteogenic cells & $6.2 \pm 1.8$ & $67.4 \pm 2.0$ & $8.0 \pm 1.6$ & $6.9 \pm 1.2$ & $14.2 \pm 1.5$ & $14.3 \pm 1.7$ \\
\hline Skin & $3.2 \pm 0.6$ & $3.7 \pm 0.7$ & $3.4 \pm 0.6$ & $3.0 \pm 0.5$ & $6.6 \pm 0.6$ & $6.7 \pm 0.6$ \\
\hline Spleen & $4.4 \pm 0.8$ & $5.2 \pm 0.9$ & $5.4 \pm 1.0$ & $4.7 \pm 0.8$ & $9.8 \pm 0.9$ & $9.9 \pm 0.9$ \\
\hline Thymus & $4.8 \pm 0.9$ & $5.7 \pm 1.0$ & $6.4 \pm 1.2$ & $5.6 \pm 1.0$ & $11.2 \pm 1.0$ & $11.3 \pm 1.0$ \\
\hline Thyroid & $4.2 \pm 0.8$ & $4.4 \pm 0.8$ & $8.3 \pm 1.4$ & $7.3 \pm 1.3$ & $12.4 \pm 1.2$ & $11.7 \pm 1.1$ \\
\hline Urinary bladder wall & $59.1 \pm 5.2$ & $79.5 \pm 6.7$ & $5.5 \pm 1.0$ & $4.8 \pm 0.8$ & $64.6 \pm 4.8$ & $84.3 \pm 6.4$ \\
\hline Uterus & - & $8.8 \pm 1.4$ & - & $4.6 \pm 0.8$ & - & $13.4 \pm 1.2$ \\
\hline Effective dose $^{d}$ & $8.1 \pm 1.2$ & $10.1 \pm 1.4$ & $5.3 \pm 1.0$ & $4.6 \pm 0.8$ & $13.4 \pm 1.1$ & $14.7 \pm 1.2$ \\
\hline
\end{tabular}

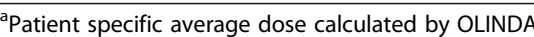

${ }^{\mathrm{b}}$ Patient specific average dose calculated by ImPACT

'Ovaries for female patients/Testes for male patients

${ }^{\mathrm{d}}$ Effective dose in $\mathrm{mSv}$ estimated by ICRP Publication 103 
Table 4 Organ equivalent dose and effective dose from 18F-FDG PET/CT with diagnostic CT to adult male and female patients

\begin{tabular}{|c|c|c|c|c|c|c|}
\hline \multirow[t]{2}{*}{ Organ } & \multicolumn{2}{|c|}{ Dose from $18 \mathrm{~F}-\mathrm{FDG}^{\mathrm{a}}$} & \multicolumn{2}{|c|}{ Dose from $\mathrm{CT}^{\mathrm{b}}$} & \multicolumn{2}{|l|}{ Total dose } \\
\hline & Male $(n=49)$ & Female $(n=49)$ & Male $(n=49)$ & Female $(n=49)$ & Male $(n=49)$ & Female $(n=49)$ \\
\hline Adrenals & $5.0 \pm 0.6$ & $5.9 \pm 1.1$ & $17.6 \pm 3.7$ & $13.5 \pm 5.5$ & $22.6 \pm 3.4$ & $19.4 \pm 5.0$ \\
\hline Brain & $15.9 \pm 2.1$ & $16.6 \pm 3.6$ & $21.0 \pm 4.4$ & $16.1 \pm 6.5$ & $36.9 \pm 3.6$ & $32.8 \pm 5.5$ \\
\hline Breasts & $3.6 \pm 0.5$ & $4.2 \pm 0.9$ & $15.7 \pm 3.3$ & $12.0 \pm 4.8$ & $19.3 \pm 3.0$ & $16.2 \pm 4.4$ \\
\hline Gallbladder wall & $5.4 \pm 0.7$ & $6.0 \pm 1.2$ & $18.7 \pm 3.9$ & $14.4 \pm 5.8$ & $24.1 \pm 3.1$ & $20.4 \pm 5.2$ \\
\hline Colon & $4.9 \pm 0.6$ & $5.8 \pm 1.1$ & $17.5 \pm 3.7$ & $13.5 \pm 5.5$ & $22.4 \pm 3.4$ & $19.3 \pm 5.0$ \\
\hline Small intestine & $5.1 \pm 0.6$ & $5.6 \pm 1.1$ & $17.5 \pm 3.7$ & $13.5 \pm 5.5$ & $22.6 \pm 3.4$ & $19.1 \pm 5.0$ \\
\hline Stomach wall & $4.7 \pm 0.6$ & $5.4 \pm 1.1$ & $18.6 \pm 3.9$ & $14.4 \pm 5.8$ & $23.3 \pm 3.6$ & $19.8 \pm 5.3$ \\
\hline Heart wall & $29.0 \pm 4.1$ & $34.4 \pm 7.7$ & $20.0 \pm 4.2$ & $15.3 \pm 6.2$ & $48.9 \pm 3.8$ & $49.7 \pm 6.4$ \\
\hline Kidneys & $4.4 \pm 0.5$ & $5.2 \pm 1.0$ & $19.1 \pm 4.0$ & $14.7 \pm 6.0$ & $23.5 \pm 3.8$ & $19.9 \pm 5.5$ \\
\hline Liver & $8.9 \pm 1.2$ & $10.7 \pm 2.2$ & $17.9 \pm 3.8$ & $13.8 \pm 5.6$ & $26.8 \pm 3.3$ & $24.5 \pm 4.7$ \\
\hline Lungs & $8.3 \pm 1.1$ & $9.8 \pm 2.1$ & $20.0 \pm 4.2$ & $15.3 \pm 6.2$ & $28.3 \pm 3.7$ & $25.1 \pm 5.2$ \\
\hline Muscle & $4.2 \pm 0.5$ & $4.8 \pm 1.0$ & $12.5 \pm 2.7$ & $9.6 \pm 3.9$ & $16.7 \pm 2.4$ & $14.4 \pm 3.4$ \\
\hline Ovaries/Testes ${ }^{c}$ & $4.6 \pm 0.6$ & $6.8 \pm 1.3$ & $3.5 \pm 0.7$ & $12.6 \pm 5.1$ & $8.1 \pm 0.6$ & $19.4 \pm 4.5$ \\
\hline Pancreas & $5.2 \pm 0.6$ & $6.1 \pm 1.2$ & $17.2 \pm 3.6$ & $13.2 \pm 5.3$ & $22.4 \pm 3.3$ & $19.3 \pm 4.8$ \\
\hline Red marrow & $4.1 \pm 0.6$ & $4.7 \pm 1.1$ & $14.6 \pm 3.0$ & $11.2 \pm 4.5$ & $18.7 \pm 2.7$ & $15.9 \pm 3.9$ \\
\hline Osteogenic cells & $6.1 \pm 1.2$ & $7.1 \pm 2.1$ & $26.4 \pm 5.5$ & $20.3 \pm 8.2$ & $32.6 \pm 4.8$ & $27.5 \pm 7.0$ \\
\hline Skin & $3.2 \pm 0.4$ & $3.6 \pm 0.7$ & $11.1 \pm 2.3$ & $8.6 \pm 3.4$ & $14.2 \pm 2.1$ & $12.2 \pm 3.1$ \\
\hline Spleen & $5.2 \pm 1.08$ & $5.1 \pm 1.0$ & $17.4 \pm 3.7$ & $13.4 \pm 5.4$ & $21.7 \pm 3.4$ & $18.5 \pm 4.9$ \\
\hline Thymus & $4.4 \pm 0.5$ & $5.5 \pm 1.1$ & $21.8 \pm 4.6$ & $16.9 \pm 6.8$ & $26.6 \pm 4.3$ & $22.5 \pm 6.2$ \\
\hline Thyroid & $4.2 \pm 0.5$ & $4.3 \pm 0.9$ & $28.0 \pm 5.8$ & $21.6 \pm 8.7$ & $32.2 \pm 5.6$ & $25.9 \pm 8.2$ \\
\hline Urinary bladder wall & $59.1 \pm 3.7$ & $78.9 \pm 9.2$ & $18.5 \pm 3.9$ & $14.2 \pm 5.7$ & $77.7 \pm 4.8$ & $93.1 \pm 10.2$ \\
\hline Uterus & - & $8.6 \pm 1.6$ & - & $13.8 \pm 5.8$ & - & $22.4 \pm 4.9$ \\
\hline Effective dose ${ }^{d}$ & $8.1 \pm 0.8$ & $9.9 \pm 1.6$ & $17.4 \pm 3.7$ & $13.4 \pm 5.5$ & $25.5 \pm 3.4$ & $23.4 \pm 4.9$ \\
\hline
\end{tabular}

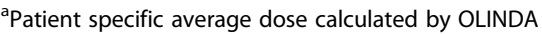

b Patient specific average dose calculated by ImPACT

'Ovaries for female patients/Testes for male patients

${ }^{\mathrm{d} E f f e c t i v e}$ dose estimated by ICRP publication 103

and brain. CT organ equivalent doses are summarized in Tables 3 and 4 .

The five organs with the highest organ equivalent doses from diagnostic CT in male patients, in order of highest to lowest dose, were thyroid, osteogenic cells, thymus, brain and lungs, and in female patients thyroid, osteogenic cells, thymus, brain and heart.

\section{Radiation doses - total dose}

The mean $\pm \mathrm{SD}$ (range) effective dose from the combined PET and CT portions of all PET/CT exams was $19.6 \pm 6.1 \mathrm{mSv}$ (11.0 to $34.3 \mathrm{mSv}$ ) for all patients,
$19.5 \pm 2.3 \mathrm{mSv}$ (11.0 to $31.1 \mathrm{mSv}$ ) for male patients and $19.1 \pm 3.1 \mathrm{mSv}$ (12.8 to $34.3 \mathrm{mSv}$ ) for female patients.

The mean \pm SD (range) effective dose from the combined PET and CT portions of the exams using standard CT was $14.0 \pm 1.3 \mathrm{mSv}$ (11.0 to $17.6 \mathrm{mSv}$ ) for all patients, $13.4 \pm 1.1 \mathrm{mSv}$ (11.0 to $16.2 \mathrm{mSv}$ ) for male patients and $14.7 \pm 1.2 \mathrm{mSv}$ (12.8 to $17.6 \mathrm{mSv}$ ) for female patients.

The mean \pm SD (range) total effective dose from the combined PET and CT portions of the exams using diagnostic CT technique was $24.4 \pm 4.3 \mathrm{mSv}$ (14.6 to

Table 5 18F-FDG Dose summary

\begin{tabular}{|c|c|c|c|c|c|c|}
\hline \multirow[t]{2}{*}{$\mathrm{CT}$ technique description } & \multicolumn{2}{|c|}{ Injection activity (MBq) } & \multicolumn{2}{|c|}{ Effective dose (mSv) } & \multicolumn{2}{|c|}{ Effective dose per unit activity (mSv/MBq) } \\
\hline & Male & Female & Male & Female & Male & Female \\
\hline Standard CT & $455 \pm 30$ & $450 \pm 26$ & $8.1 \pm 1.2$ & $10.1 \pm 1.4$ & $0.018 \pm 0.002$ & $0.022 \pm 0.002$ \\
\hline Diagnostic $C T$ & $455 \pm 25$ & $454 \pm 51$ & $8.1 \pm 0.8$ & $9.9 \pm 1.6$ & $0.018 \pm 0.002$ & $0.022 \pm 0.003$ \\
\hline
\end{tabular}

Data are the mean \pm standard deviation. $n$, male standard $C T=46 . n$, female standard $C T=39 . n$, male diagnostic $C T=49 . n$, female diagnostic $C T=49$ 
Table $6 \mathrm{CT}$ effective dose summary for all patients

\begin{tabular}{lllllr}
\hline $\begin{array}{l}\mathrm{CT} \\
\text { technique } \\
\text { description }\end{array}$ & $\begin{array}{l}\mathrm{DLP} \\
(\mathrm{mGy} \mathrm{cm})\end{array}$ & $\begin{array}{l}\mathrm{mAs} \\
(\mathrm{mAs})\end{array}$ & $\begin{array}{l}\text { Effective dose } \\
(\mathrm{mSv})\end{array}$ & $\begin{array}{l}\text { Effective dose per unit mAs } \\
(\mathrm{mSv} / \mathrm{mAs})\end{array}$ & $\begin{array}{l}\text { Effective dose per unit DLP } \\
(\mathrm{mSv} / \mathrm{mG} \mathrm{cm})\end{array}$ \\
\hline Standard & $438 \pm 84.8$ & $39 \pm 11$ & $7.9 \pm 1.5$ & $0.134 \pm 0.028$ & $0.013 \pm 0.002$ \\
Diagnostic & $1050 \pm 342$ & $119 \pm 51$ & $15.4 \pm 5.0$ & $0.142 \pm 0.056$ & $0.015 \pm 0.0002$ \\
\hline
\end{tabular}

Data are the mean \pm standard deviation

$34.3 \mathrm{mSv}$ ) for all patients, $25.5 \pm 3.4 \mathrm{mSv}$ (17.8 to $31.1 \mathrm{mSv}$ ) for male patients and $23.4 \pm 4.9 \mathrm{mSv}$ (14.6 to $34.3 \mathrm{mSv}$ ) for female patients. Total doses are summarized in Tables 7 and 8 .

The five organs with the highest organ equivalent doses from PET/CT in all patients, in order of highest to lowest dose, were bladder, heart, brain, liver and lungs. PET/CT organ equivalent doses are summarized in Tables 3 and 4.

\section{Discussion}

Risk from radiation exposure to the individual patient is important for evaluation of risk/benefit for justification, and relevant to protocol optimization and personnel protection [40]. The doses themselves utilized in the risk estimation are useful for characterizing the patient population and image acquisition practices of a clinic. A dose estimation method can be based on a combination of reference values and patient- and scanner-specific data. Accurate estimation of patient dose, rather than a conservatively high estimate, is important for many oncology patients who can potentially have many scans and must track cumulative radiation exposure. The accuracy of the patient dose used in the estimation of risk depends on the dose estimation method employed: dose estimated based on reference values depends on the characteristics of the patient population in comparison with the reference population, while the accuracy of the dose estimated based on specific information depends on the extent to which specific information is utilized. Exam- and patient-specific factors are accounted for in an ideal approach to dose estimation. In the current study, we assessed radiation dosimetry of PET/CT for all organs using patient- and exam-specific data and commonly available dosimetry resources.

Effective dose is commonly used when evaluating relative biologic risk, and the Monte Carlo-based organ dose coefficients should not be used to calculate effective dose for individual patients $[6,8]$. The International Organization for Medical Physics, ICRP, Health Physics Society and American Association of Physicists in Medicine (AAPM) all have issued policy statements that note the dangers of extrapolating biological risk estimates for radiation doses less than $100 \mathrm{mSv}$ [6, 9-11]. The establishment and use of risk coefficients to estimate public health determinants from individual or population exposures must be considered in the context of uncertainties in the estimates [12]. These uncertainties include dosimetric uncertainties, epidemiological and methodological uncertainties, uncertainties from low statistical power and precision in epidemiology studies of radiation risk, uncertainties in modeling risk data, generalization of risk estimates across different populations and dose rates, as well as reliance on epidemiological studies on observational rather than experimental data [13]. Uncertainties in such risk estimates have been suggested as being up to a factor of 3 lower or higher than the value itself [14]. These rather large uncertainties cause predictions of radiation induced cancers or detriment to be susceptible to biases and confounding influences that are unidentifiable. With due recognition of the limitations imposed by the uncertainties inherent in correlating dose with risk, effective dose is a useful approximation for relating patient population characteristics and evaluating optimization efforts in addition to the clinical benefits of exams.

For internal absorbed dose assessment, OLINDA\EXM was used to perform dosimetry calculations for the various body organs [32]. This code allows calculations for 814 radionuclides and a wide variety of adult, pediatric and pregnant female phantoms; furthermore, it also allows users to modify organ masses in the phantoms for more patient-specific dose calculations. Organ doses calculated by OLINDA $\backslash$ EXM based on reference phantoms

Table 7 Effective dose for male and female patients

\begin{tabular}{|c|c|c|c|c|c|c|}
\hline \multirow[t]{2}{*}{$\begin{array}{l}\text { CT Technique } \\
\text { Description }\end{array}$} & \multicolumn{2}{|c|}{$\begin{array}{l}\text { 18F-FDG PET } \\
(\mathrm{mSV})\end{array}$} & \multicolumn{2}{|l|}{$\begin{array}{l}\mathrm{CT} \\
(\mathrm{mSv})\end{array}$} & \multicolumn{2}{|l|}{$\begin{array}{l}\text { Total } \\
\text { (mSv) }\end{array}$} \\
\hline & Male & Female & Male & Female & Male & Female \\
\hline Standard CT & $8.1 \pm 1.2$ & $10.1 \pm 1.4$ & $5.3 \pm 1.0$ & $4.6 \pm 0.8$ & $13.4 \pm 1.1$ & $14.7 \pm 1.2$ \\
\hline Diagnostic CT & $8.1 \pm 0.8$ & $9.9 \pm 1.6$ & $17.4 . \pm 3.7$ & $13.4 \pm 5.5$ & $25.5 \pm 3.4$ & $23.4 \pm 4.9$ \\
\hline
\end{tabular}

Data are the mean \pm standard deviation 
Table 8 Effective dose summary for all patients

\begin{tabular}{lcrl}
\hline CT technique description & 18F-FDG PET & \multicolumn{1}{c}{ CT } & Total \\
\hline Standard CT & $9.0 \pm 1.6$ & $5.0 \pm 1.0$ & $14.0 \pm 1.3$ \\
Diagnostic CT & $9.0 \pm 1.6$ & $15.4 \pm 5.0$ & $24.4 \pm 4.3$ \\
\hline
\end{tabular}

Data are the mean \pm standard deviation in $\mathrm{mSv}$

representing the average patient were found to be in good agreement with patient specific Monte Carlo mean dose estimates [41]. Monte Carlo results suggest that the difference between the organ equivalent dose estimates in our calculations and those derived from the use of earlier stylized MIRD-type phantoms can be different by a margin as great as $277 \%[42]$. Patient-specific organ masses were derived from previous reports on variations in the mass of different body organs in relation to stature and BMI ([3739]. Marine et al, and Clark et al described phantoms that model different body types in a series of percentile height phantoms to evaluate the variation of specific absorbed fractions with height and weight differences across the human population $[37,39]$. With patient-specific data and a mean injected activity of $454 \mathrm{MBq}$, the mean effective dose of $9.0 \mathrm{mSv}$ is consistent with dose per unit injected activity reported in the literature, and also with the dose estimates from ICRP publication 106 [23, 25-28, 34]. The relatively higher injected activity in our clinic at the time of the study results in a relatively higher whole body dose than is observed in other clinics with lower fixed standard injection activity or body mass adjusted injection activity. Despite a lack of 18F-FDG uptake in lung, this organ received the third-highest organ dose according to our methods, and this is most likely due to the proximity of the lung to the heart. Injected activity, patient body mass and height, all available from the patient record, were among the specific factors employed in our estimation of specific internal dose from 18F-FDG.

ImPACT Dose is a readily available CT organ dose calculation tool that reports organ doses based on modeled radiation transport data specific to the CT scanner and represents improved accuracy over previous methods [17]. Without using patient-specific inputs to ImPACT, organ dose was overestimated by $17 \%$. A contributing factor to this difference is likely the relatively larger size of our patient population. The mean BMI of our patient population was 26.4 while a reference BMI from ICRP Reference Man characteristics is 23.5. It should be noted that there is an opportunity to make the CT organ dose estimate more specific than in our study. Because the Dose Length Product (DLP) in the exam dose report is itself a general value based on a single phantom, the dose estimate may be made more specific by modifying CTDIvol to account for patient size [43]. Scannerspecific results such as the average scan length are useful for identifying opportunities for optimization and for evaluation against similar protocols and standards. For example, the scan length for a whole-body PET/CT can be compared to the scan length for a whole body $\mathrm{CT}$ at the same clinic or to a reference value. The average \pm SD scan length of diagnostic whole body PET/CT in the present study is $89.5 \pm 14.7 \mathrm{~cm}$, while the combined scan length of chest + abdomen + pelvis CT scans is reported as $73 \mathrm{~cm}$ [29]. Knowledge of the relative scan lengths puts differences in dose and DLP in perspective. Further, the anatomy in a diagnostic PET/CT is fixed to PET anatomy selection, while the anatomy during the CTonly acquisition may be optimally adjusted. Anatomy selection, and avoidance, is an important aspect of CT protocol optimization that may be overlooked for incorporation into PET/CT scanners. Individual technologists may incorporate optimal anatomy selection in both CT and PET/CT scans, but this optimization measure is more likely to be routinely incorporated as a matter of policy for CT scanners than PET/CT scanners utilized for diagnostic PET/CT exams. In these ways, scanner-specific data are useful for characterizing the clinic's practices and identifying opportunities for optimization.

Although the average diagnostic PET/CT scan length in this study is greater than that reported in the literature, the dose from the CT portion of the diagnostic $\mathrm{PET} / \mathrm{CT}$ exams we evaluated in this study is consistent with the average whole body CT dose reported in the literature. Table 9 summarizes doses reported in the literature for whole body $\mathrm{CT}$ and chest-abdomen-pelvis (CAP) $\mathrm{CT}$ exams, which have approximately the same length scans as whole body PET/CT scans [22, 24, 29-31].

The average value of the five included studies reporting whole-body or equivalent CT dose is $15.1 \mathrm{mSv}$ and likely represents a conservative estimate of average dose from a whole body diagnostic CT. A clear advantage of a combined diagnostic PET/CT is the higher patient throughput relative to separate scheduling, along with a relatively shorter exam time than the lower-dose alternatives. From the patient's perspective, a single visit to the imaging clinic is easier than two separate visits. If the doses are the same, then a combined scan has the additional patient benefit convenience. Depending on the contributing factors at a given imaging clinic, the radiation dose from a standard PET/CT with separate

Table 9 Comparison of reported whole body diagnostic CT effective dose

\begin{tabular}{llllllll}
\hline CT study & $1^{\mathrm{a}}$ & $2^{\mathrm{b}}$ & $3^{\mathrm{c}}$ & $4^{\mathrm{d}}$ & $5^{\mathrm{e}}$ & Average & This study \\
\hline Effective dose (mSv) & 19 & 9.25 & 12.5 & 22.5 & 16 & 15.1 & 15.4
\end{tabular}

Data are mean $\mathrm{mSv}$

${ }^{\mathrm{a}} \mathrm{CT}$-based transmission scan in high quality mode [31]

${ }^{\mathrm{b}} 73-\mathrm{cm}$ CAP CT [29]

'Whole body CT, C3 to symphisis pubis [22]

${ }^{\mathrm{d} C o m p l e t e}$ whole body CT includes thyroid contribution [24]

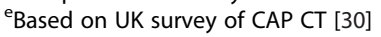


diagnostic $\mathrm{CT}$ may be more or less than the radiation dose from a diagnostic PET/CT. Intuitively, a PET/CT with diagnostic technique compared to a standard PET/CT with separately acquired diagnostic CT may appear to be qualitatively equivalent but dosimetrically different because the PET/CT with diagnostic technique CT can also be used for attenuation correction. CT dose from the standard PET/CT appears to be in addition to the dose from the separate diagnostic CT. However, differences in $\mathrm{PET} / \mathrm{CT}$ and $\mathrm{CT}$ scanner hardware and software can cause the dose to be higher or lower. For example, the number of slices is typically different between dual- and singlemodality units, and adaptive filtering may not available on PET/CT machines. Both hardware and software factors can cause the patient dose from a combined scan to be higher or lower than separately acquired scans and must be considered when evaluating the dose at a given clinic with reference or literature doses. Less variation is observed in reported PET dose that is estimated from a single source, such as ICRP 106. Even the standards at an imaging clinic for uptake time and acquisition time can affect dose by influencing injected activity. A busy clinic with a fixed injected activity per patient, for example, may administer relatively more activity to account for the inevitable changes in scheduling that come with patient cancellations, delays and other impacts on timing. There is evidence, however, that weight- or BMI-adjusted injected activity reduces patient dose and staff exposure without compromising image quality [44]. A busy clinic may also benefit from a relatively higher injected activity to shorten the acquisition time, as PET acquisition is based on total counts in a bed field and more activity results in the total counts achieved relatively sooner.

The potential for variation in reported dose from PET is apparent in the variability in those PET exam parameters that depend on clinical setting. The patient dose in a clinic employing Germanium-based attenuation correction will differ from a clinic that uses CT-based attenuation correction. Germanium-based attenuation correction has been found to result in negligible patient dose, but it also results in lower diagnostic value in terms of anatomical delineation and longer scanning time than CT-based attenuation correction [31]. The additional challenge of coregistering separate image sets also accompanies this approach. Nonetheless, Germanium-based attenuation correction is sufficient for standard PET/CT exams and still finds routine usage amongst clinics that wish to add PET imaging, but already have a CT scanner. The possibility of different attenuation correction methods makes a one-size-fits-all estimate of expected radiation dose for PET/CT exams unrealistic, and promotes a specific approach to dose evaluation.

Brix et al evaluated total dose from PET/CT exams utilizing CT-based attenuation correction at different hospitals and demonstrated that regardless of whether the PET/CT protocol is standard or diagnostic, the dose from a PET/CT exam is about $23 \mathrm{mSv}$, due to different approaches, hardware, standards and clinical objectives [23]. RPOP reference doses are compared with the results of our study and with typical literature data in Table 10.

The range of doses observed in Table 10 demonstrates the importance of careful choice of data, characterizing the dose at one's own clinic and understanding the factors contributing to patient dose from PET/CT.

Dose estimated using reference values and standard body models requires the least amount of effort and results in a non-specific estimate that may not be consistent with the characteristics of the actual patient population. However, many exam-specific parameters are conveniently accessed with minimal effort. Reference DLP values are available, but in practice the actual DLP from the exam is readily available from the dose report and is easily used to estimate effective dose by employing either a standard or scanner-specific factor [18]. An approach employing all available patient-specific resources (i.e. patient-adjusted DLP and organ mass, scanner-specific factors) results in accurate estimation but requires moderate effort because this data must be retrieved from electronic records. A retrospective dose estimate requires accessing DICOM information and patient records for relevant information, while estimating dose from a present exam for an individual patient can utilize information at hand. Whether the risk estimate is made at the time of the exam or retrospectively, and whether it is for an individual or representative group, the effort involved in gathering the information must be weighed against the value of the information gained.

\section{Conclusions}

An ideal dose estimation method is convenient to employ and considers clinic- and patient-specific factors to an appropriate extent. Patient specific parameters (e.g., weight, and height) identified from patient data allow for more accurate estimate of organ equivalent doses and the effort involved in obtaining the data is worthwhile

Table 10 Comparison of reference dose with our results and literature

\begin{tabular}{llll}
\hline & RPOP & This study & Literature $^{\text {a }}$ \\
\hline Injected activity (MBq) & 400 & 454 & 370 \\
PET dose & 8 & 9 & 7 \\
Standard CT dose & 7 & 5 & 2.7 \\
Diagnostic CT dose & 30 & 15.4 & 16.1 \\
Total dose, standard PET/CT & 15 & 14 & 9.7 \\
Total dose, Diagnostic PET/CT & 38 & 24.4 & 23.1 \\
\hline
\end{tabular}

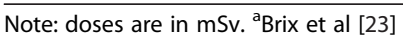


for a retrospective analysis of a representative patient sample and for some individual patients. Accurate estimation of patient dose, rather than a conservatively high estimate, is important for many oncology patients who can potentially have many scans and must track cumulative exposure. Considering actual patient-specific characteristics resulted in lower organ equivalent dose estimates than traditional reference phantoms and models, but the methods employed in this study resulted in organ doses and effective doses that are in agreement with alternative methods. Considering the substantial radiation dose of whole body PET/CT exams and the potential for wide variation in contributing factors, all aspects of the exam process should be considered for optimization. Radiopharmaceutical injection activity, CT technique, CT protocol optimization measures, anatomy selection, and convenience to both patient and imaging clinic are all factors which must be considered for comprehensive optimization and subsequent justification of the PET/CT exam. The effort involved in obtaining patient-specific data is reasonable to help justify the risk associated with the radiation exposure, optimize the image acquisition parameters to balance dose with the objectives of the exam, and characterize the patient population.

Clinic-specific patient dose for each type of PET/CT exam can reasonably be estimated for comparison with industry standards, reference values and literature values. Opportunities for optimization may also be apparent in better understanding how the clinic's doses compare with other sources of data.

\section{Abbreviations}

AAPM, American Association of Physicists in Medicine; BMI, Body mass index; ICRP, International Commission on Radiological Protection; $\mathrm{PET} / \mathrm{CT}$, Fluorine-18 positron emission tomography/computed tomography; RPOP, Radiation protection of patients

\section{Acknowledgements}

The authors wish to acknowledge the assistance of the department of Radiology and Molecular Imaging and Therapy Service for their support of the project. This work was supported by Memorial Sloan Kettering Cancer Center Support Grant/Core Grant (P30 CA008748).

\section{Availability of data and materials}

The dataset supporting the conclusions of this article is available upon request to the corresponding author

\section{Authors' contributions}

BQ drafted the manuscript and analyzed data. ZD collected and analyzed data, participated in design of the study and helped draft the manuscript. NPT provided practical clinical input and technical review of imaging parameters and helped draft the manuscript. HS provided practical and technical clinical input and technical review of imaging procedures and helped draft the manuscript. LD conceived of and designed the study, provided technical oversight for dose calculation methods and results. All authors read and approved the manuscript.

\section{Authors' information}

BQ and ZD are Medical Health Physicists, NPT and HS are nuclear medicine physicians in the molecular imaging and therapy service with clinical expertise in positron emission tomography, LD is a Medical Health Physicist with expertise in dose and risk estimation methodology.

\section{Competing interests}

The authors declare that they have no competing interests.

\section{Consent for publication}

Not applicable.

\section{Ethics approval and consent to participate}

Institutional review board approval with waiver of patient informed consent was obtained from the Memorial Sloan Kettering Cancer Center Institutional Review Board/Privacy Board.

\section{Author details}

${ }^{1}$ Department of Medical Physics, Box 84, Memorial Sloan Kettering Cancer Center, 1275 York Avenue, New York, NY 10065, USA. ${ }^{2}$ Department of Radiology, Memorial Sloan Kettering Cancer Center, New York, USA.

Received: 12 February 2016 Accepted: 2 June 2016

Published online: 18 June 2016

\section{References}

1. Czernin J, Schelbert H. PET/CT Imaging: Facts, opinions, hopes, and questions. J Nucl Med. 2004;45(Suppl):1S-3S.

2. Czernin J, Allen-Auerbach M, Schelbert HR. Improvements in cancer staging with PET/CT: Literature-based evidence as of September 2006. J Nucl Med. 2007;48(1):78S-885.

3. Bockisch A, Beyer T, Antoch G, et al. Positron emission tomography/ computed tompgraphy-imaging protocols, artifacts, and pitfalls. Mol Imag Bio. 2004;6:188-89.

4. ICRP. Radiological Protection and Safety in Medicine. A Report of the International Commission on Radiological Protection. Ann ICRP. 1996;26:1-47

5. Brink JA, Amis E. Image wisely: a campaign to increase awareness about adult radiation protection. Radiology. 2010;257(3):601-2.

6. ICRP. The 2007 Recommendations of the International Commission on Radiological Protection, ICRP Publication 103. Ann ICRP. 2007;37:1-332.

7. Stabin MG. Uncertainties in Internal Dose Calculations for Radiopharmaceuticals. J Nucl Med. 2008;119:853-60.

8. Shrimpton PC, Wall B, Yoshizumi T, Hurwitz LM, Goodman PC. Effective Dose and Dose-Length Product in CT. Radiology. 2009;250(2):604-5.

9. Hendee WR, I.O.f.M.P. Policy Statement of the International Organization for Medical Physics. Radiology. 2013;267(2):326-7.

10. Health Physics Society. Radiation Risk in Perspective. Health Physics Society. https://hps.org/documents/radiationrisk.pdf.

11. AAPM. AAPM Position Statement on Radiation Risks from Medical Imaging Procedures. PP 25-A. American Association of Physicists in Medicine. http://www. aapm.org/org/policies/details.asp?id=318\&type=PP. Accessed 7 Jun 2016.

12. Dauer LT, Branets I, Stabulas-Savage J, et al. Optimising Radiographic Bitewing Examination to Adult and Juvenile Patients Through the Use of Anthropomorphic Phantoms. Radiat Prot Dosimetry. 2013;158:51-8.

13. NCRP. Uncertainties in Internal Radiation Dose Assessment. NCRP Report 164. NCRP Publications, Bethesda, MD; 2009.

14. UNSCEAR. Report of the United Nations Scientific Committee on the Effects of Atomic Radiation. Fifty-ninth Session. General Assembly Official Records Sixty Seveth Session, Supplemental No. 46.A/67/46. United Nations Scientific Committee on the Effects of Atomic Radiation. http://www.unscear.org/docs/reports/2012/ UNSCEAR2012Report 15-08936 eBook website.pdf. Accessed 7 Jun 2016.

15. IAEA. Radiation Protection of Patients PET/CT scanning. https://rpop.iaea.org/RPOP/RPoP/Content/InformationFor/HealthProfessionals/ 6_OtherClinicalSpecialities/PETCTscan.htm. Accessed 7 Jun 2016.

16. Kamel E, Hany T, Burger $C$, et al. CT vs 68Ge Attenuation Correction in a Combined PET/CT System: Evaluation of the Effect of Lowering CT Tube Current. Eur J Nucl Med Mol Imaging. 2002;29(3):346-50.

17. Kalender WA, Schmidt B, Zankl M, Schmidt M. A PC Program for Estimating Organ Dose and Effective Dose Values in Computed Tomography. Eur Radiol. 1999:9(3):555-62.

18. Kalender W, Deak P, Smal Y. Multisection CT protocols: sex- and age-specific conversion factors used to determine effective dose from dose length product. Radiology. 2010;257(1):158-66. 
19. ICRP. Report of the Task Group on Reference Man. ICRP Publication 23. Oxford: Pergamon Press; 1975.

20. Measurements, I.C.o.R.U.a. Measurement of Dose Equivalents from External Photon and Electron Radiations. ICRU Report 47. Bethesda, MD: ICRU; 1992.

21. ICRP. Radiation Dose to Patients from Radiopharmaceuticals: Addendum 2 to ICRP Publication 53, Also Includes Addendum 1 to ICRP Publication 72. ICRP Publication 80. Oxford, UK: Pergamon Press; 1998.

22. Brenner DJ, Elliston C. Estimated Radiation Risks Potentially Associated with Full-Body CT Screening. Radiology. 2012;232:735-8.

23. Brix G, Lechel U, Glatting G, Ziegler S, Munzig W, Muller S, Beyer T. Radiation Exposured of PAtients Undergoing Whole-Body Dual-Modality 18F-FDG PET/CT Examinations. J Nucl Med. 2005;46(4):608-13.

24. Groves AM, Owen K, Courtney HM, et al. 16-detector Multiclice CT: Dosimetry Estimation by TLD Measurement Compared with Monte Carlo Simulation. Br J Radiol. 2004;77:662-5.

25. Huang B, Law M, Khong PL. Whole-Body PET/CT Scanning: Estimation of Radiation Dose and Cancer Risk. Radiology. 2009;251(1):166-74.

26. Khamwan K, Krisanachinda A, Pasawang P. The Determination of Patient Dose From F-18-FDG PET/CT Examination. Radiat Prot Dosimetry. 2010;141:50-5.

27. Mahmud $\mathrm{MH}$ et al. Estimation of patient radiation dose from whole body18F- FDG PET/CT examination in cancer imaging: a preliminary study. Journal of Physics: Conference Series. 2014:546:012008.

28. Leide-Svegborn S. Radiation Exposure of Patients and Personnel from a PET/CT Procedure with 18F-FDG. Radiat Prot Dosimetry. 2010;139:208-13.

29. Theocharopoulos N, Damilakis J, Perisinakis K, et al. Effective Doses to Adult and Pediatric Patients from Multislice Computed Tomography: A Method Based on Energy Imparted. Med Phys. 2006;33(10):3846-56.

30. Tsalafoutas IA, Koukourakis G. Patient Dose Considerations in Computed Tomography Examinations. World J Radiol. 2004;2(7):262-8.

31. Wu TH, Huang Y, Lee JJ, et al. Radiation Exposure During Transmission Measurements: Comparison Between CT- and Germanium-based Techniques with a Current PET Scanner. Eur J Nucl Med Mol Imaging. 2004;77:662-5.

32. Stabin MG, Sparks R, Crowe E. OLINDA/EXM: The Second-Generation Personal computer Software for Internal Dose Assessment in Nuclear Medicine. J Nucl Med. 2005;46(6):1023-7.

33. Bolch WE, Eckerman K, Sgouros G, Thomas SR. MIRD Pamphlet No 21: A Generalized Schema for Radiopharmaceutical Dosimetry-Standardization of Nomenclature. J Nucl Med. 2009;50(3):477-84.

34. ICRP. Radiation Dose to Patients from Radiopharmaceuticals. Addendum 3 to ICRP Publication 53. ICRP Publication 106. Ann ICRP. 2008;38(1-2):1-197.

35. Hays MT, Watson E, Thomas SR, Stabin M. MIRD Dose Estimate Report No 19: Radiation Absorbed Dose Estimates from 18F-FDG. J Nucl Med. 2002; 43(2):210-4

36. Deloar HM, Fujiwara T, Shidahara M, Nakamura T, Watabe H, Narita Y, Itoh M, Miyake M, Watanuki S. Estimation of Absorbed Dose for 2-[F-18]fluoro-2deoxy-D-glucose Using Whole-Body Positron Emission Tomography and Magnetic Resonance Imaging. Eur J Nucl Med. 1998;25(6):585-74.

37. Clark LD, Stabin M, Fernald MJ, Brill AB. Changes in Radiation Dose with Variations in Human Anatomy: Moderately and Severely Obese Adults. J Nucl Med. 2010;51:929-32.

38. ICRP. Basic Anatomical and Physiological Data for Use in Radiological Protection: Reference Values. A Report of Age- and Gender-Related Differences in the Anatomical and Physiological Characteristics of Reference Individuals. Ann ICRP. 2002:32:5-265.

39. Marine PM, Stabin MG, Fernald MJ, Brill AB. Changes in Radiation Dose with Variations in Human Anatomy: Larger and Smaller Normal-Stature Adults. J Nucl Med. 2010;51(5):806-11.

40. ICRP. ICRP Publication 105. Radiation Protection in Medicine. Ann ICRP. 2007;37:1-63

41. Grimes J, Celler A. Comparison of Internal Dose Estimates Obtained Using Organ-Level, Voxel 5 Value, and Monte Carlo Techniques. Med Phys. 2014;41(9):92501.

42. Ding A, Gu J, Liu H, Caracappa P, Xu XG. The Design of a New PC Software for Estimating Patient Doses from CT Scans. Health Phys. 2009;97(1):S56.

43. AAPM. Size-specific dose estimates (SSDE) in pediatric and adult body CT examinations: Report of AAPM Task Group 204. College Park, MD: American Association of Physicists in Medicine; 2011.

44. Saade C, H.M., Ammous A et al, Weight-based Protocols during Whole Body FDG PET/CT Significantly Reduces Radiation Dose without Compromising Image Quality, in RSNA 2015 Scientific Assembly and Annual Meeting. Chicago, IL.

\section{Submit your next manuscript to BioMed Central and we will help you at every step:}

- We accept pre-submission inquiries

- Our selector tool helps you to find the most relevant journal

- We provide round the clock customer support

- Convenient online submission

- Thorough peer review

- Inclusion in PubMed and all major indexing services

- Maximum visibility for your research

Submit your manuscript at www.biomedcentral.com/submit
) Biomed Central 